\title{
Application of PSO Algorithm in Parameter Optimization of Biodegradable Medical Polymer Degradation Model
}

\author{
Zhang Ying ${ }^{\mathrm{a}}$, Zhang Tao-hong, ${ }^{\mathrm{a},}$, Xin Rui-wu ${ }^{\mathrm{b}}$, Yang Bing-ru ${ }^{\mathrm{a}}$ \\ ${ }^{a}$ School of Information Engineering, University of Science \& Technology Beijing, Beijing, China \\ ${ }^{b}$ Jurong Ontuo Technology Co. Ltd., Jurong, China
}

\begin{abstract}
The particle swarm optimization (PSO) algorithm is a stochastic global optimization technique based on swarm intelligence. It possesses advantages such as being a simple principle, few parameters and easy to be realized. In this paper, an optimization model is established to solve the difficulty in selecting parameters and improve the simulation accuracy of the biodegradable medical polymer degradation model. When modeling, the particle swarm optimization (PSO) algorithm is proposed to solve the model and calculate undetermined parameters of the biodegradable medical polymer degradation equations. A comparative analysis of the calculation results is progressed. It shows that parameters determined by optimization model make the simulation results of degradation model more close to the experiment results. Using this method to solve the model is more accurate and efficiency than determining parameters artificially. It also shows that the particle swarm optimization algorithm used to optimize parameters have practical significance and application value.
\end{abstract}

Index Terms: biodegradable medical polymer material degradation equations; particle swarm optimization algorithm; parameters optimization

(C) 2011 Published by MECS Publisher. Selection and/or peer review under responsibility of the Research Association of Modern Education and Computer Science.

\section{Introduction}

Biodegradable medical polymer materials, typically poly (lactide) (PLA) poly (glycolide) (PGA of various form) and their copolymers are currently being used in the human body as fixation devices in the form of screws, pins, meshes, etc., to protect the healing of fractured bones. These polymers have different degradation rates and mechanical properties. But now the determination of parameters in degradation equations is still depended on artificial implementation. It possesses shortcomings such as great difficulty in work, poor rationality, low efficiency and heavy burden. As improving the parameters' reliability of the

* Corresponding author.

E-mail address: waterswordzth@163.com 
degradation equations becomes increasingly prominent, people have been trying to search for a reliable and effective method to solve this problem.

In this paper, we abstract a suitable mathematical optimization model based on an overall consideration of the actual principles in degradation of polymer materials and various factors. The optimization scheme for parameters' determination in degradation equations is provided by Particle Swarm Optimization. It avoids the inadequacy of artificial determining process, improves the safety and reliability of parameters, identifies more reasonable parameter values and makes simulation results of experimental model become better.

\section{Establishment of degradation equations' parameter optimization model}

\subsection{Polymer degradation equations}

At present the study of the biodegradation process of medical polymer materials is focused on chemical hydrolysis of the polymer backbone. This phase includes four stages: (a) water absorption, (b) fracture of the ester bonds, (c) diffusion of water-soluble oligomer and (d) dissolution of fragments. First is water absorption, small water molecules contacting the surface of polymer and diffusing into the ester bonds or around hydrophilic groups. Under the influence of acid or alkali medium, ester bonds in macromolecular main chain generate free hydrolysis and convert the long chains into shorter fragments. Then it causes the sample's number average molecular weight decreases slowly and loses its mechanical strength. When molecular weight is small enough to the water-soluble limit value, the overall structure starts to be deformed and loss in weight and the sample dissolves subsequently, generating soluble degradation products.

The biodegradation process is modelled using a set of simplified reaction-diffusion equations. However, there has been very limited amount of work in the literature on mathematical modelling of biodegradation. Most of the published papers are in the context of controlled drug release, almost all of which are for very simple geometries [1-7]. In 2008, Pan et al. [8,9] published a general basic model for the degradation of medical polymer materials in Biomaterials. The model is firstly used to solve for simple cases of plates and pins. Specific degradation model is as follows:

$$
\left\{\begin{array}{l}
\frac{\mathrm{d} \bar{C}_{\mathrm{e}}}{\mathrm{d} \bar{t}}=-\left(\bar{k}_{1} C_{\mathrm{e}}+\bar{C}_{\mathrm{e}} \bar{C}_{\mathrm{m}}^{\mathrm{n}}\right) \\
\frac{\mathrm{d} \overline{C_{m}}}{\mathrm{~d} \bar{t}}=\bar{k}_{1} \bar{C}_{\mathrm{e}}+\bar{C}_{\mathrm{e}} \bar{C}_{\mathrm{m}}^{\mathrm{n}}+\bar{D}_{\mathrm{o}} \operatorname{div}_{\bar{x}_{i}}\left\{\left[1+\alpha\left(1-\bar{C}_{\mathrm{m}}-\bar{C}_{\mathrm{e}}\right)\right] \operatorname{grad}_{\bar{x}_{i}}\left(\bar{C}_{\mathrm{m}}\right)\right\}
\end{array}\right.
$$

By above knowable, these partial differential equations are non-dimensionalised giving two normalised parameters which control the interplay between the hydrolysis reaction and the monomer diffusion. These formulas demonstrate the weight of biodegradable polymer molecular changes with degradation time. There are three parameters in the model still need to be determined by manual setting, namely $\bar{k}_{1}, \bar{D}_{0}$ and a, which are referred to the degradation rate, the diffusion coefficient and the diffusion factor.

\subsection{Establishment of parameters optimization model}

The degradation model of biodegradable polymer materials simulates the degradation process of polymer materials by computer and the simulation results should be consistent with the experimental data. Therefore, the objective function of the optimization is setting as:

$$
\min \sum_{i}\left|\bar{C}_{\mathrm{e}}^{*}(i)-\bar{C}_{\mathrm{e}}(i)\right|
$$

in which $\bar{C}_{\mathrm{e}}^{*}(i)$ represents the concentration of ester bonds calculated by the model, $\bar{C}_{\mathrm{e}}(i)$ is experimental value of the concentration of ester bonds and $i$ is degradation time(week). The objective function is intended 
to minimize the cumulative absolute errors between calculated values and experimental values of the concentration of ester bonds during degradation time.

The optimizing parameters of this parameter optimization model are $\bar{k}_{1}, \bar{D}_{0}$ and a. The constraint conditions of the optimization model are as follows:

$$
\left\{\begin{array}{l}
\frac{\mathrm{d} \bar{C}_{\mathrm{e}}}{\mathrm{d} \bar{t}}=-\left(\bar{k}_{1} C_{\mathrm{e}}+\bar{C}_{\mathrm{e}} \bar{C}_{\mathrm{m}}^{\mathrm{n}}\right) \\
\frac{\mathrm{d} \bar{C}_{m}}{\mathrm{~d} \bar{t}}=\bar{k}_{1} \bar{C}_{\mathrm{e}}+\bar{C}_{\mathrm{e}} \bar{C}_{\mathrm{m}}^{\mathrm{n}}+\bar{D}_{0} \underset{\bar{x}_{i}}{\operatorname{div}}\left\{\left[1+\alpha\left(1-\bar{C}_{\mathrm{m}}-\bar{C}_{\mathrm{e}}\right)\right] \underset{\bar{x}_{i}}{\left.\operatorname{grad}\left(\bar{C}_{\mathrm{m}}\right)\right\}}\right. \\
0 \leq \bar{k}_{1} \leq 2 \\
0 \leq \bar{D}_{0} \leq 20 \\
0 \leq a \leq 10
\end{array}\right.
$$

The purpose to design this parameter optimization model is to make the values of $\bar{C}_{\mathrm{e}}^{*}(i)$ calculated by the degradation model more close to the experiment result $\bar{C}_{\mathrm{e}}(i)$ through optimizing the three parameters. It makes up shortcomings such as great difficulty in work, poor rationality, low efficiency and heavy burden, which are caused by manual setting parameters.

\subsection{Optimization model of PSO algorithm}

Particle swarm optimization (PSO algorithm) is a swarm intelligence-based optimization algorithm proposed by Kennedy and Eberhart in 1995[10, 11]. The algorithm regards each individual of colony as a particle possessing flight direction and speed in the D-dimensional searching space. In the iteration process, each particle continuously makes statistics for optimal values of itself and the colony to adjust its flight direction and speed. In this process, the individual gradually migrates to the better region and eventually makes colony search to the problem's optimal solution. The migration of particles could be performed by the following equations:

$$
\begin{aligned}
& v_{i d}(k+1)=\omega v_{i d}(k)+c_{1} r_{1}\left[p_{i d}(k)-x_{i d}(k)\right]+c_{2} r_{2}\left[p_{g d}(k)-x_{g d}(k)\right] \\
& x_{i d}(k+1)=x_{i d}(k)+v_{i d}(k+1)
\end{aligned}
$$

where $\mathrm{i}=1,2 \ldots \mathrm{m}, \mathrm{m}$ represents the total number of particles in the colony, $\mathrm{d}=1,2 \ldots \mathrm{D}, \mathrm{d}$ is the dimension in the solution space, $\mathrm{X}_{\mathrm{i}}=\left(\mathrm{x}_{\mathrm{i} 1}, \mathrm{x}_{\mathrm{i} 2} \ldots, \mathrm{x}_{\mathrm{iD}}\right)^{\mathrm{T}}$ is the coordinate of the ith particle which means where the particle locates at, $\mathrm{P}_{\mathrm{i}}=\left(\mathrm{p}_{\mathrm{i} 1}, \mathrm{p}_{\mathrm{i} 2}, \ldots, \mathrm{p}_{\mathrm{iD}}\right)^{\mathrm{T}}$ represents the best position of the ith particle, $\mathrm{P}_{\mathrm{g}}=\left(\mathrm{p}_{\mathrm{g} 1}, \mathrm{p}_{\mathrm{g} 2}, \ldots \mathrm{p}_{\mathrm{gD}}\right)^{\mathrm{T}}$ represents the best position of the colony searched by all the particles, $V_{i}=\left(v_{i 1}, v_{i 2}, \ldots v_{i D}\right)^{T}$ is the ith particle's flying velocity, $\mathrm{k}$ is the iterative times, $\mathrm{c}_{1}$ and $\mathrm{c}_{2}$ are two accelerating factors, $\mathrm{r}_{1}$ and $\mathrm{r}_{2}$ are generated randomly in the interval $[0,1], \omega$ represents the inertia weighting factor which is nonnegative to coordinate global and local optimizing capacity of particle swarm.

According to the degradation parameter optimization model we propose, the position of each particle $\mathrm{X}_{\mathrm{i}}(\mathrm{t})=\left(\mathrm{x}_{\mathrm{i} 1}(\mathrm{t}), \mathrm{x}_{\mathrm{i} 2}(\mathrm{t}), \mathrm{x}_{\mathrm{i} 3}(\mathrm{t})\right)$ is a potential solution of the optimization problem. Suppose that $\mathrm{x}_{\mathrm{i} 1}(\mathrm{t}), \mathrm{x}_{\mathrm{i} 2}(\mathrm{t})$ and $\mathrm{x}_{\mathrm{i} 3}(\mathrm{t})$ represent $\bar{k}_{1}, \bar{D}_{0}$ and $\alpha$, whose particle positions range corresponding to [0,2], [0,20], [0, 10], respectively.

The number of particles $\mathrm{m}$ is set to 20 , iteration number $\mathrm{k}$ is set to 21 , accelerating factors $\mathrm{c}_{1}$ and $\mathrm{c}_{2}$ are both set to 2 , the value of inertia weighting factor $\omega$ linear decreases from 0.9 to 0.4 as iteration goes on and the value of objective function takes as fitness. Initially, we randomly initialize position and velocity of particles as $\mathrm{X}_{\mathrm{i}}(1)=\left(\mathrm{x}_{\mathrm{i} 1}(1), \mathrm{x}_{\mathrm{i} 2}(1), \mathrm{x}_{\mathrm{i} 3}(1)\right)$ and $\mathrm{V}_{\mathrm{i}}(1)=\left(\mathrm{v}_{\mathrm{i} 1}(1), \mathrm{v}_{\mathrm{i} 2}(1), \mathrm{v}_{\mathrm{i} 3}(1)\right)$, then optimize parameters according to the formula (5) and (6) iteratively. 


\section{Calculation results and discussion}

This paper takes Visual $\mathrm{C}++6.0$ as a tool to develop the design of optimal program in the Windows platform completely. Furthermore, the program is used to solve optimization parameters of the degradation equations' optimization model. When using particle swarm optimization algorithm to optimize the model, the initial solution is completely randomly generated. Table 1 demonstrates parameters' values and values of objective function (fitness value) solved by using particle swarm optimization algorithm during the 21 times iterative process. It is visible that the optimal parameters are obtained at the 20th iteration. Subsequently, the optimal parameters are brought into (1) and (2) to solve the polymer molecular weight $\bar{C}_{\mathrm{e}}^{*}(i)$ and then the results are contrasted.

There are 25 sets of data to compare with the weights of polymer molecular calculated by optimization model. The contrast data are experimental data obtained via doing degradable experiment with the plates made of DLPLA materials by Grizzi et al. [12] and data calculated by using manual setting parameters when Pan's model built [8], respectively. The comparison result is shown in Fig. 1.

Particle swarm optimization is used in this paper to establish optimization model of the degradation equations. We optimize the parameters of the polymer materials' degradation optimization model using the particle swarm optimization algorithm and compare the optimized calculation with Pan's calculation. Experiment results show that absolute errors of predicted values obtained from optimal parameters by using the particle swarm optimization algorithm is 0.229391 , which is lower than the model before optimization about $49.93 \%$. It shows that the particle swarm optimization algorithm achieves the optimization purposes, which makes up shortcomings caused by manual setting parameters, improves the reliability of the parameters, makes designed optimization plans more reasonable, reduces the calculation error and advances the accuracy of the experiment.

Table 1 Parameter values and objective function values of PSO's iterative process

\begin{tabular}{|l|l|l|l|l|}
\hline Iterative times & \multicolumn{1}{|c|}{$\bar{k}_{1}$} & \multicolumn{1}{c|}{$\boldsymbol{\alpha}$} & \multicolumn{1}{c|}{$\bar{D}_{0}$} & \multicolumn{1}{c|}{ Fitvalue } \\
\hline 1 & 0.681173 & 9.56755 & 2.05573 & 0.312005 \\
\hline 2 & 0.669838 & 9.50078 & 10.404 & 0.238711 \\
\hline 3 & 0.659645 & 9.52648 & 7.09072 & 0.237568 \\
\hline 4 & 0.659645 & 9.52648 & 7.09072 & 0.237568 \\
\hline 5 & 0.660592 & 9.36914 & 6.6847 & 0.235663 \\
\hline 6 & 0.661718 & 9.12641 & 4.86844 & 0.23232 \\
\hline 7 & 0.661718 & 9.12641 & 4.86844 & 0.23232 \\
\hline 8 & 0.656279 & 8.58924 & 4.37006 & 0.231812 \\
\hline 9 & 0.62769 & 9.18219 & 2.5786 & 0.230062 \\
\hline 10 & 0.62769 & 9.18219 & 2.5786 & 0.230062 \\
\hline 11 & 0.62769 & 9.18219 & 2.5786 & 0.230062 \\
\hline 12 & 0.62769 & 9.18219 & 2.5786 & 0.230062 \\
\hline 13 & 0.62769 & 9.18219 & 2.5786 & 0.230062 \\
\hline 14 & 0.62769 & 9.18219 & 2.5786 & 0.230062 \\
\hline 15 & 0.62769 & 9.18219 & 2.5786 & 0.230062 \\
\hline 16 & 0.62769 & 9.18219 & 2.5786 & 0.230062 \\
\hline 17 & 0.64014 & 9.01548 & 3.24709 & 0.229434 \\
\hline 18 & 0.64014 & 9.01548 & 3.24709 & 0.229434 \\
\hline 19 & 0.64014 & 9.01548 & 3.24709 & 0.229434 \\
\hline 20 & 0.634784 & 9.63779 & 2.62336 & 0.229391 \\
\hline 21 & 0.634784 & 9.63779 & 2.62336 & 0.229391 \\
\hline
\end{tabular}




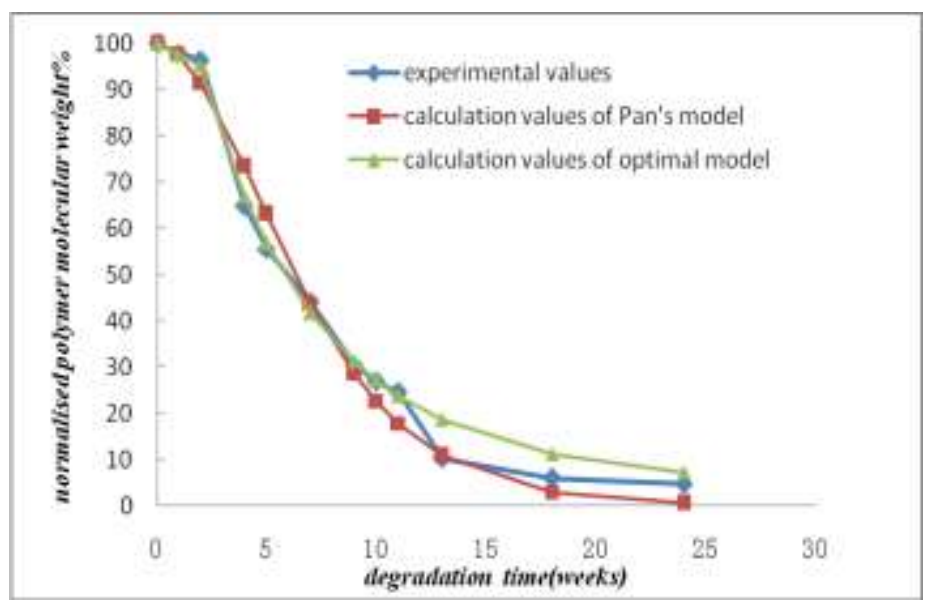

Fig. 1 Contrast of optimal results

\section{References}

[1] Siepmann J, Peppas NA, "Modelling of drug release from delivery systems based on hydroxypropyl methylcellulose (HPMC)," in Advanced Drug Delivery Reviews, vol. 48, 2001, pp. 139-57.

[2] Joshi A, Himmelstein KJ, "Dynamics of controlled release from bioerodible matrices," in Journal of Controlled Release, vol. 15, 1991, pp. 95-104.

[3] Zygourakis K, Markenscoff PA, "Computer-aided design of bioerodible devices with optimal release characteristics: a cellular automata approach," in Biomaterials, vol. 17, 1996, pp. 125-35.

[4] Göpferich A, Langer R, "Modelling monomer release from bioerodible polymers ," in Journal of Controlled Release, vol. 33, 1995, pp. 55-69.

[5] Siepmann J, Siepmann F, Florence AT, “ Local controlled drug delivery to the brain: mathematical modelling of the underlying mass transport mechanisms ," in International Journal of Pharmaceutics, 2006, pp. $101-19$.

[6] Yu Ruixia, CHEN Hualing, Chen Tianning, "CA-based biodegradable drug delivery system modeling and simulation," in System Simulation, 2007, pp. 183-186. (in Chinese)

[7] Wang Xiaopeng, Chen Tianning, Yang Zhanxiao, "Monte Carlo model based on micro-PLGA delivery systems modeling and simulation," in Actuators, vol. 19, 2006, pp. 1327-1329. (in Chinese)

[8] Wang Y, Pan JZ, Han XX, Csaba Sinka, Lifeng Ding, "A phenomenological model for the degradation of biodegradable polymersin," in Biomaterials, vol. 29, 2008, pp. 3393-3401.

[9] Han XX, Pan JZ, "A model for simultaneous crystallisation and biodegradation of biodegradable polymers," in Biomaterials, vol. 30, 2009, pp. 423-430.

[10] Kennedy J., Eberhart R.C., "Particle Swarm Optimization," Proceedings of the 1995 IEEE International Conference on Neural Networks, Piscataway, NJ, Perth, Australia: IEEE service center, 1995, pp. 19421948.

[11] Eberhart R.C., Kennedy J, “A new optimizer using Particle Swarm Theory, ” Proceedings of the Sixth International Symposium on Micro Machine and Human Science, Nagoya, Japan, 1995, pp. 39-43.

[12] Grizzi I, Garreau H, Li S, Vert M, "Hydrolytic degradation of devices based on poly[DL- lactic acid] size dependence," in Biomaterials, vol. 16, 1995, pp. 305-311. 